\title{
A 20-year-old Lady with Peritoneal Tuberculosis Presented as Acute Abdomen and A Review of Peritoneal Tuberculosis
}

\author{
Shahiduzzaman GKM' ${ }^{1}$, Tahmina A ${ }^{2}$, Kabir CMS $^{3}$, Mohsena A ${ }^{4}$ Zaman RA $^{5}$, Siddique ${ }^{6}$
}

Conflict of Interest: None Received: 16 July 2016 Accepted: 10 September 2016 www.banglajol.info/index.php/JSSMC

Key Words:

Peritoneal tuberculosis, acute abdomen, AFB

\begin{abstract}
:
Tuberculosis can entail any component of the gastrointestinaltract and is the sixth most common site of extra pulmonaryassociation. Both the incidence and severity ofabdominal tuberculosis are predictable to amplify withgrowingnumbers of HIV infection. Peritoneal tuberculosis, a type of abdominal tuberculosis, occurs in three forms: wet typewith ascites, dry type with adhesions, and fibrotic type withomental thickening and loculated ascites. Clinically, peritoneal tuberculosis ischaracterized by fever, abdominal pain, anorexia, weightloss, and ascites. Nevertheless, not any of these symptoms is accuratefor the disease, so it is frequently misdiagnosed, especiallyas carcinomatous peritonitis in the elderly. Diagnosisnear the beginning of peritoneal tuberculosis is of keyimpact in the control of thedisease. Chest X-rays demonstratesupport of associated pulmonarylesions in less than 25\% of cases. Laparoscopywith direct biopsy is an exceptionalinvestigativetechniqueand should be considered for each patient with unsolvedascites. A classicconclusion requires detection ofbacilli in ascitic fluid or peritoneum tissue. However, acidfaststaining is frequently negative and cultures are positivein $30-40 \%$ of cases, making bacteriological evidence ofthe disease extremely difficult. In recent times, advances in moleculartechniques have provided aninnovative approach to the fast diagnosisof tuberculosis by nucleic acid probes and polymerasechain reaction. But if molecular techniques fail or unavailable, then presence of caseating granuloma in biopsy material is accepted as hallmark of extra-pulmonary tuberculosis and as significant as positive Acid Fast Bacillus (AFB) in pulmonary tuberculosis. Management is with conventionalanti-tubercular treatment for at least six months.
\end{abstract}

Keywords: Peritoneal tuberculosis, acute abdomen, $A F B$

[J Shaheed Suhrawardy Med Coll 2016; 8(2): 63-68] DOI: http://dx.doi.org/10.3329/jssmc.v8i2.37246

\section{Introduction:}

Tuberculosis was a prevalent infection even in Ancient Greece and Egypt. The disease was put underneath control only after the introduction of antimicrobial therapy in 1946. Autopsies conducted on patients with pulmonary tuberculosis prior to the period of effective anti-tubercular

1. Dr. GKM Shahiduzzaman, Professor, Department of Medicine, ShSMCH, Dhaka.

2. Dr. Tahmin Akhter, MO, Department of Virology, BSMMU, Dhaka.

3. Dr. C. M. Shamim Kabir, IMO, Department of Medicine, ShSMCH, Dhaka.

4. Dr. Mohsena Akter, HMO, Department of Medicine, ShSMCH, Dhaka.

5. Dr. Roksana AkhterZaman, IMO, Department of Medicine, ShSMCH, Dhaka.

6. Dr. Shahjahan Siddique, IMO, Department of Medicine, ShSMCH, Dhaka.

Correspondence to: Dr. GKM Shahiduzzaman, Professor, Department of Medicine, ShSMCH, Dhaka. drugs discovered intestinal involvement in $55-90 \%$ cases, with the frequency interrelated to the extent of pulmonary involvement. ${ }^{1}$ Pimparkar et al. found proof of abdominal tuberculosis (bowel, peritoneum and liver) in 3.72 per cent of 11,746 autopsies carried out in K.E.M. Hospital, Mumbai between 1964 to $1974 .^{2}$ Following a few decades of declining numbers of tuberculosis, throughout the most recent 10 years there has been a global reemergence of the disease. Thismay possibly be due to numerous reasons such as the HIV epidemic, an increase in the quantity of immigrants and the primary resistance to first-line drugs. One-third of the world population is in danger of acquiring tuberculosis according to World Health Organization and more than 30 million deaths due to tuberculosis were estimated in the nineties, especially in Africa and Asia. ${ }^{3}$ Not unexpectedly, there is furthermore an increase in the percentage of patients with atypical presentations and atypical extra-pulmonary forms of tuberculosis. Extra- 
pulmonary organ involvement of tuberculosis is estimated as $10-15 \%$ of patients not infected with HIV whereas the frequency is about $50-70 \%$ in patients infected with HIV. ${ }^{4}$ Abdominal tuberculosis is one of the most ubiquitous forms of extra pulmonary infection. Peritoneal tuberculosis is a form of abdominal tuberculosis that involves the omentum, parietal and visceral peritoneum of intestinal tract, liver, spleen, or female genital tract. It accounts for about $1-2 \%$ of all cases of tuberculosis. Owing to the imprecise course of the disease there are enormous difficulties in its diagnosis. Different methods of investigation have been reported as gold standards; though, there are great difficulties in medical practice. As a consequence, the diagnosis of peritoneal tuberculosis is at a standstill a challenge to the clinician.

\section{Case:}

Mrs. Nasima of 20 years, married, normotensive, non diabetic woman from Kishoreganj was admitted in our hospital with the complaints of fever for one \&a half month and painful abdominal swelling for the same duration. Fever was insidious in onset, low grade, intermittent, evening rise and was not associated with chills and rigor and did not subside with sweating. Maximum temperature was not recorded. This fever was not connected with any cough, chest pain, joint ache, oral ulceration, loose motion, skin rash, photosensitivity, alopecia and jaundice. Moreover, she noticed abdominal pain and swelling for same period. At first pain was insidious, dull aching over right lumbar and right iliac regions but within a week it became intermittent and colicky involving the right iliac, right lumbar and extending up to the epigastric region. Pain was moderate in intensity and became worse after taking food, journey by bus or rickshaw, during defecation $\&$ felt better to some extent by compressing over the area by pillow or by hand.

Furthermore, patient developed abdominal swelling that was also insidious in onset and gradually progressive but her urine habit was normal. She had noticeable anorexia but no vomiting and had constipation. For the previous 2 days prior to admission she developed high-grade fever, severe abdominal colicky pain and further deteriorating abdominal swelling. Fever was high grade, continuous, not associated with chills and rigor and subsided for 1-2 hours after taking tablet paracetamol. Maximum-recorded temperature was $104^{\circ} \mathrm{F}$.Abdominal pain was severe, colicky, intermittent and started at right iliac region. After 3-4 hours it radiated to entire abdomen but there was no vomiting. Pain was not relieved after defecation. In addition,she observed more abdominal swelling throughout these two days period.
On examination patient was ill looking, agitated, mildly anemic, Pulse $120 / \mathrm{min}$ and BP 100/60 mm-Hg. Temperature was $104^{\circ} \mathrm{F}$, respiratory rate $20 / \mathrm{min}$. On systemic examination abdomen was distended, flanks were full, movement of abdomen was restricted with respiration. Umbilicus was centrally placed and inverted. There was no visible peristalsis or engorged vein. There was diffuse severe tenderness over the entire abdomen. Owing to severe tenderness patient did not allow deep palpation. Ascites was present as evidenced by shifting dullness. Examination of other system revealed no abnormality.

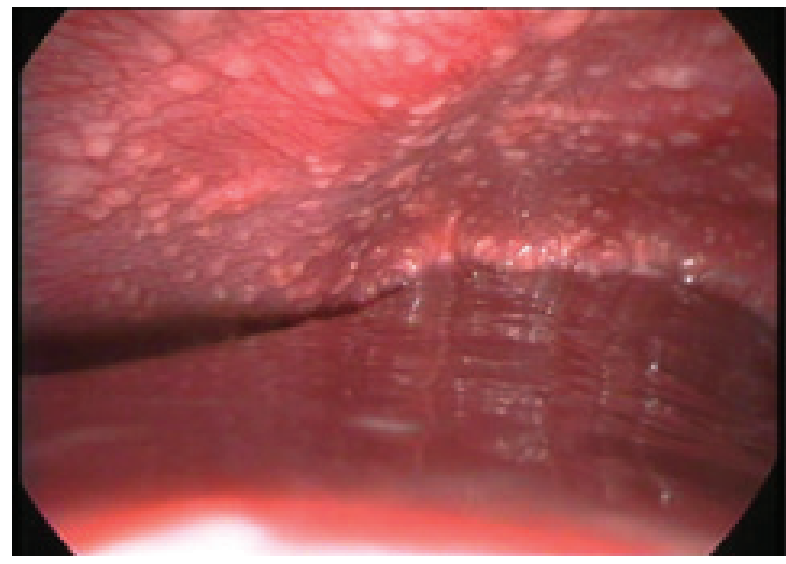

Fig.-1: Laparoscopic view of abdomen showing parietal and visceral peritoneum are studded with numerous sago like pearls characteristics of peritoneal tuberculosis.

She underwent routine and specific investigations. Her complete blood count showed Hb. $12.10 \mathrm{gm} / \mathrm{dl}$, TC 10,430/ cmm, DC: N-74\%, L-16\%, Platelet 510,000/cmm and ESR 54 $\mathrm{mm}$ in $1^{\text {st }}$ hour. Urine $\mathrm{M} / \mathrm{E}$ reveals epithelial cells $18-20$ / HPF, RBC 12-16/HPF (patient was on menstruation). There was no growth of organism in urine culture. Her renal and liver functions tests were normal and serum lipase level 28 $\mathrm{U} / \mathrm{L}(\mathrm{N}<100 \mathrm{U} / \mathrm{L})$. Viral markers including HBV, HCV \& HIV $1 \& 2$ were negative. ANA showed titer of 17.81 LPU. Mantoux testwas positive with an induration of $25 \mathrm{~mm}$.

Her CXR and Plain X-ray abdomen showed normal findings. Her ECG showed sinus tachycardia and echocardiography was normal. USG (W/A) was done twice, 10 days apart. First one reported moderate to marked ascites, while the second one reported moderate ascites.

Ascitic fluid tap was done which was straw in color and samples were sent for acid-fast bacilli (AFB) smear and culture for M. tuberculosis, Grams stain, biochemistry and cytology. Total count of WBC was $40 / \mathrm{cmm}$ and differential count showed 100\% lymphocytes.AFB was negative. Cytology was negative for malignant cells. Ascitic fluid protein was $4.9 \mathrm{gm} / \mathrm{dl}$ and sugar was $82.8 \mathrm{mg} / \mathrm{dl}$. Ascitic fluid adenosine deaminase (ADA) was $72.30 \mathrm{U} / \mathrm{L}(\mathrm{N}<30)$. Her colonoscopy report was normal. 
In spite of adequate supportive and conservative treatment of acute abdomen, there was no improvement. Laparoscopic examination of abdomen was planned. Laparoscopy was done and 6 pieces of greyish white tissues (largest one $2 \mathrm{mmin}$ diameter) were collected and sent for histopathology. Laparoscopic view of abdomen showed parietal and visceral peritoneums were studded with sago like pearls.Histopathology report confirmed granulation tissue, infiltration of inflammatory cells, epithelioid granuloma, multinucleated giant cells, area of necrosis, suggestive of tissue from tubercular abscess wall. There was no evidence of malignancy. Finally, it leads to conclusion of peritoneal tuberculosis (wet type).

After 4 months, patient came for a follow up visit. Her temperature was normal, no abdominal pain, distension or ascites. There was progressive improvement and weight gain.

\section{Pathogenesis:}

Peritoneal tuberculosis is generally associated with a primary focus of tuberculosis somewhere else. This primary focus is typically the lung, on the other hand only about one third of cases have clinical or radiographic confirmation of pulmonary tuberculosis. Like in other forms of extra-pulmonary tuberculosis, smaller number of bacilli than those found in pulmonary disease is responsible for much greater damage. In addition, the paucity of bacilli may be united with a fairly unapproachable or unfamiliarsite of extra-pulmonary tuberculosis infection, making laboratory evidence extremely difficult.The postulated mechanisms by which the tuberclebacilli are able to gain entrance to the peritoneal cavity are: transmurallyfrom diseased bowel, throughout lymph channelsfrom infected abdominal lymph nodes, from tuberculous salpingitis, or else, more commonly, by hematogenous spreadfrom a pulmonary focus. ${ }^{5,6} \mathrm{In}$ peritoneal tuberculosis, the peritoneum is studded with numerous sago pearl like tubercles. It is thick and hyperemic with a loss of its glistening luster. The omentum is also thickened. Strictures may result from cicatrical healing of circumferential tubercular ulcers. Occlusive arterial changes mayturn out ischemia and also add to the development of strictures. ${ }^{7-10}$ Mesenteric lymph nodes can be enlarged, matted and may caseate. Distinctive granulomas may be seen just in the mesenteric lymph nodes. This is particularly frequent in patients who have taken anti-tubercular treatment for some time. The reverse, i.e., the existence of granulomas in the intestine and no granulomas in the draining lymph nodes is uncommon. ${ }^{11}$ Peritoneal tuberculosis occurs in three forms: (1) wet type with ascites, (2) encysted (loculated) type with a localized abdominal swelling; and (3) fibrotic type with abdominal masses composed of mesenteric and omental thickening, with matted bowel loops felt as lumps in the abdomen. A mixture of these types is also common. ${ }^{1}$

\section{Clinical features:}

Normally the onset is relatively insidious, with more than $70 \%$ of patients having had symptoms for more than 4 months prior to ultimate conclusion. The most regular symptoms are constitutional and comprise fever, anorexia, weakness, malaise, and weight loss. Abdominal distention caused whichever by ascites or by partial obstruction may be present. On examination, the abdomen is diffusely tender in most of the patients; yet, the characteristic doughy abdomen is hardly ever found. Peritoneal tuberculosis should be suspected in high-risk or immunocompromised patients with ascites, fever, unexplained general symptoms, and diffuses abdominal pain or tenderness. ${ }^{12}$

\section{Diagnosis:}

Usual laboratory and radiographic studies are hardly everdiagnostic. A typical leukocyte count is present in the majority of patients, and anemia is just variably found. Tuberculin skin tests are frequently positive in patients with tuberculous peritonitis; conversely a negative outcome is of no assistancein excluding the disease. ${ }^{13}$ Radiographs of the abdomenare rarely of benefit; but, a CT-scan may be helpfulin identifying thickened bowel and ascites. ${ }^{14}$ The diagnosis of tuberculous peritonitis is often recommended by result at laparoscopy or laparotomy. ${ }^{15}$ Tuberculous peritonitis is characterized by stalactitelikefibrinous masses from the parietal peritoneum and inaddition, may be studded with tiny granulomas. The differential diagnosis of tuberculous peritonitisis unpredictable, depending on the severity of the symptoms. In patients with a long-drawn-out history, tuberculous peritonitisis most frequently confused with Crohn's disease orcarcinoma. In patients presenting acutely, the differential diagnoses should take account of such entities as acute appendicitis, cholecystitis, perforated ulcer and salpingitis.

\section{Ascitic fluid examination:}

Examination of the peritoneal fluid may provide useful evidence.In patients with tuberculous peritonitis, the ascitic fluid is straw colored with protein $>3 \mathrm{~g} / \mathrm{dl}$, and entire cellcount of $150-4000 / \mathrm{cmm}$, consisting chiefly of lymphocytes $(>70 \%)$. The ascites to blood glucose proportion isless than 0.96 and serum ascites albumin gradient is lessthan $1.1 \mathrm{~g} / \mathrm{dl}$. The yield of organisms on smear and culture is low.Staining for acid fast bacilli is 
positive in less than $3 \%$ ofcases. A positive culture is obtained in less than $30 \%$ ofcases, and it takes 6-8 weeks for the mycobacterial coloniesto appear. ${ }^{16,17}$ However, in aprevious study, cultures set subsequent to centrifugation of 1 liter of ascitic fluid showed $83 \%$ positivity. ${ }^{18}$ Adenosine deaminase (ADA) is an aminohydrolase that converts adenosine to inosine and is thus involved in the catabolism of purine bases. The enzyme activity ismore in T than in B-lymphocytes, and is proportional to the degree of T cell differentiation. ADA is increased in tuberculous ascitic fluid due to stimulation of T-cells by mycobacterial antigens. ADA levels were determined in the ascitic fluid of 49 patients by Dwivedi et al. the levels in tuberculous ascites were not ablyel evated than thosein cirrhotic or malignant ascites. ${ }^{19}$ Taking a cut off level of $33 \mathrm{U} / 1$, the sensitivity, specificity and diagnostic accuracywere 100, 97 and 98\% correspondingly. In coinfection with HIV, the ADA values can be usual or low down. Misleadinglyhigh values can happen in malignant ascites. High interferon-levels in tubercular ascites have been reported to be helpful diagnostically. Combination of both ADA and interferon estimations may promote enhance sensitivity. ${ }^{20,21}$

\section{Molecular techniques:}

In current years, advances in molecular techniques have provided a latest approach to the fast diagnosis of tuberculosis by nucleic acid probes and $\mathrm{PCR}^{22}$.The insertion sequence IS6110 has been fruitfully used as atarget for PCR amplification in clinical samples by a lot of investigators. The sensitivity and specificity of IS6110 amplification is uneven in different laboratories and depends on the supply of the clinical sample, the localization of the tuberculosis, the coexistence of HIV infection, and other technical parameters. DNA amplification of Mycobacterium tuberculosis does not always indicate viable bacilli, and the PCR result has to be evaluated in combination with other clinical and laboratory result. Lastly, response to treatment constitutes the perfect decisive factor for the diagnosis of peritoneal tuberculosis and confirms the outcome of molecular study.

\section{Ultrasonography:}

Ultrasonography can be remarkably of use for imaging peritoneal tuberculsis. ${ }^{22}$ The following features may be seen, generally in combination. ${ }^{23}$ (1) Intra-abdominal fluid, which may be free or loculated; and clear or complex (with debri and septae). Fluid collections in the pelvis may have thick septa and can mimic ovarian cyst. (2) Club sandwich or sliced bread sign is due to localized fluid between radially oriented bowel loops, due to confined exudation from the inflamed bowel (interloop ascites). (3)
Lymphadenopathy may be discrete or conglomerated (matted). The echotexture is mixed heterogeneous, in compare to the homogenously hypo echoicnodes of lymphoma. Small discrete anechoic areas representing zones of caseation may be seen insidethe nodes. With treatment the nodes show a temporary increase in size for 3-4 week and then slowly reducing size. Calcification in healing lesions is seen as discrete reflexive lines. Both caseation and calcification are very much indicative of a tubercular etiology, neither being common in malignancy associated lymphadenopathy. (4) Bowel wall thickening, best appreciated in the ileocaecal region.

\section{Computed tomographic scan:}

Tubercular ascitic fluid is of elevated attenuation value(25$45 \mathrm{HU})$ due to its high protein content. Strands, fineseptae and debris in the fluid are distinctive, but are better appreciated on ultrasonography. Thickened peritoneum and enhancing peritoneal nodules may be seen. ${ }^{24}$ Mesenteric disease on CT scan is seen as a patchy or diffuse increase in thickness, strands within the mesentery, and a stellate form. Lymph nodes may be interspersed. Omental thickening is frequently seen as an omental cake appearance. A fibrous wall, called the omental line, can cover up the omentum, developing from long standing inflammation. An omental line is not as much of common in malignant infiltration. ${ }^{25}$ Caseating lymph nodes are seen as having hypodense centers and peripheral rim enhancement. Along with calcification, these findings are very suggestive of tuberculosis. In tuberculosis the mesenteric, mesenteric root, coeliac, porta hepatis and peripancreatic nodes are characteristically involved, reflecting the lymphatic drainage of the small bowel. The retroperitoneal nodes are quite spared, and are approximately by no means seen in isolation, unlike lymphoma. ${ }^{25}$

\section{Laparoscopic findings:}

Bhargava et al. studied 87 patients with elevated ascites protein, of which 38 were diagnosed as having tuberculosis. ${ }^{26}$ They establish visual appearances to be more supportive (95\% accurate) than either histology, culture or guineapig inoculation (82, 3 and $37.5 \%$ sensitivity in that order). Caseating granulomas may be found in $85-90 \%$ of the biopsies. The laparoscopic findings in peritoneal tuberculosiscan be grouped into three categories :(1) Thickened peritoneum with tubercles: multiple, yellowish-white, uniform sized (about 4-5mm) tubercles diffusely distributed on the parietal peritoneum. The peritoneum is thickened, hyperemic and lacks its normal glistening luster. The omentum, liver and spleen can also be studded with tubercles.(2) Thickened 
peritoneum without tubercles.(3) Fibro-adhesive peritonitis with markedly thickened peritoneum and various thick adhesions fixing the viscera.

\section{Treatment:}

1. Before the introduction of chemotherapy, the mortality of tuberculous peritonitis was as high as $60 \%$; at present, the disease is, for the most part, readily curable with the on hand agents. Every patient should be given conventional antituberculous therapy for at least 6 months with first 2 months of rifampicin, isoniazid, pyrazinamide and ethambutol. A randomized comparison of six-month short course chemotherapy with a 12-month course of ethambutol and isoniazid (addition with streptomycin for the initial $2 \mathrm{wks}$ ) was conducted at the Tuberculosis Research Center, Chennai, in 193 adult patients. Curerate was 99 and $94 \%$ in patients given short-course and the 12 month regimen correspondingly. Still many physicians lengthen the treatment period from 12 to 18 months. ${ }^{27}$ The recommended surgical treatment now-a-days is conservative. ${ }^{1}$ A period of pre-operative drug therapy is controversial. Strictures, which decrease the lumen by half or more and which cause proximal hypertrophy or dilatation, are treated by strictureplasty. This involves a $5-6 \mathrm{~cm}$ long incision along the antimesenteric side, which isclosed transversely in two layers. A segment of bowel bearing numerous strictures or a single long tubular stricture may merit resection. Resection is segmental with a $5 \mathrm{~cm}$ margin. Two reports recommend that obstructing intestinal lesions may reduce with antituberculous drugs only with no surgery. Anand et al reported clinical and radiological resolution of tuberculous strictures with drug therapy even in patients with sub-acute intestinal obstruction. ${ }^{28}$ They treated 39 patients with obstructive symptoms with medical therapy. At the end of one year $91 \%$ showed clinical progress, $70 \%$ had absolute radiological resolution and surgery was required in only 3 cases ( $8 \%$ ). Predictors of necessity for surgery were long strictures $(>12 \mathrm{~cm})$ and several areas of involvement. Alike observations were made by Balasubramanianet et al. ${ }^{29}$ The mean time essential for the relief of obstructive symptoms was 6 months. The most troublesome characteristic of the resurgence of tuberculosis has been the outbreaks of multidrugresistant isolates that often fail to respond to both isoniazid and rifampicin, the two cornerstone antituberculous drugs. ${ }^{30}$ However, data from a recent study indicate that multidrug-resistant tuberculosis is a curable disease, provided that an appropriate approach to control is implemented and that appropriate treatment protocols with second line drugs are used. ${ }^{31}$

\section{References:}

1. Sharma MP, Bhatia V. Abdominal tuberculosis. Indian JMed Res 2004; 120: 305-315.

2. Pimparkar BD. Abdominal tuberculosis. J Assoc PhysiciansIndia 1977; 25: 801-811.

3. WHO. Tuberculosis control and research strategies forthe 1990ís.Memorandum from a WHO-meeting. BullWHO 1992; 70:17.

4. Runyon BA. Approach to the patient with ascites. In Textbookof Gastroenterology. T. Yamada et al editors. J B LippincottCompany, Philadelphia 2nd edition.1995: 928929.

5. American Thoracic Society Workshop. Rapid diagnostic tests for tuberculosis: what is the appropriate use? Am JRespirCrit Care Med 1997; 155: 1804-1814.

6. Uygur-Bayramicli O, Dabak G, Dabak R. A clinical dilemma:abdominal tuberculosis. World J Gastroenterol2003; 9: 1098-1101.

7. Uzunkoy A, Harma M, Harma M. Diagnosis of abdominaltuberculosis: Experience from 11 cases and review ofthe literature. World J Gastroenterol 2004; 10: 3647-3549.

8. Hopewell PC. Impact of human immunodeficiency virusinfection on the epidemiology, clinical features, managementand control of tuberculosis.Clin Infect Dis 1992;15: 540 .

9. Kapoor VK. Abdominal tuberculosis. Postgrad Med J1998; 74: 456-459.

10. Shah P, Ramakantan R. Role of vasculitis in the naturalhistory of abdominal tuberculosis $\tilde{n}$ evaluation by mesentericangiography. Indian J Gastroenterol 1991; 10:127130 .

11. Hoon JR, Dockerty MB, Pemberton J. Ileocaecal tuberculosisincluding a comparison of this disease with nonspecificregional enterocolitis and noncaseous tubercular enterocolitis. IntAbstrSurg 1950; 91: 417-440.

12. Evers BM. Diseases of the Peritoneum. In Textbook of Gastroenterology. T. Yamada et al editors. J B LippincottCompany, Philadelphia 2nd edition.1995; 103: 2308-2309.

13. Hopewell PC. Overview of clinical tuberculosis. In: BloomBR, editor, Tuberculosis. Pathogenesis, protection andcontrol, 1st edi, Washington, DC: American Society forMicrobiology; 1994, pp. 25-46, chapter 3.

14. Demirkazik FB, Akhan O, Ozmen MN, et al. US and CTfindings in the diagnosis of tuberculous peritonitis. Actaradiol 1996; 37: 517-520.

15. Apaydin B, Pakosy M, Bilir M, et al. Value of diagnosticlaparoscopy in tuberculous peritonitis. Eur J Surg 1999;165: 158-163.

16. Singh MM, Bhargava AN, Jain KP. Tuberculous peritonitis:an evaluation of pathogenetic mechanisms, diagnosticprocedures and therapeutic measures. N Engl J Med1969; 28: 10911094.

17. Marshall JB. Tuberculosis of the gastrointestinal tract andperitoneum. Am J Gastroenterol 1993; 88: 989-999. 
18. Manohar A, Simjee AE, Haffejjee AA, et al. Symptomsand investigative findings in 145 patients with tuberculousperitonitis diagnosed by peritoneoscopy and biopsyover a five year period. Gut 1990; 31: 1130-1132.

19. Dwivendi M, Misra SP, Misra V, et al. Value of adenosinedeaminase estimation in the diagnosis of tuberculousascites. Am J Gastroenterol 1990; 85: 1123-1125.

20. Hillebrand DJ, Runyon BA, Yasmineh WG, et al. Asciticfluid adenosine deamidase insensitivity in detecting tuberculousperitonitis in the United States. Hepatology1996; 24: 1408-1412.

21. Sathar MA, Simjer AE, Coovadia YM, et al. Ascitic fluidgamma interferon concentrations and adenosine deaminaseactivity in tuberculous peritonitis. Gut 1995; 36: 419-421.

22. Ritis KT, zoanopoulos D, Speletas M, et al. Amplificationof IS6110 sequence of $M$. tuberculosis complex inHIV negative patients with Fever of Unknown Origin(FUO) and evidence of extra-pulmonary disease. J InternMed 2000; 248: 415424 .

23. Jain R, Sawhney S, Bhargawa DK, et al. Diagnosis of abdominaltuberculosis: Sonographic findings in patientswith early disease. AJR 1995; 165: 1391-1195.

24. Gulati MS, Sarma D, Paul SB. CT appearances in abdominaltuberculosis. A pictorial assay.Clin Imaging 1999;23: 51-59.

25. Ha HK, Jung JI, Lee MS, et al. CT differentiation of tubercular peritonitis and peritoneal carcinomatosis. AmJ Roentgenol 1996; 167: 743-748.

26. Bhargawa DK, Shriniwas S, Chopra P, et al. Peritonealtuberculosis: Laparoscopic patterns and its diagnostic accuracy.Am J Gastroenterol 1992; 87: 109-112.

27. Balasubramanian R, Nagarajan M, Balambal R, et al. Randomizedcontrolled clinical trial of short course chemotherapyin abdominal tuberculosis: a five-year report. IntJ Tuberc Lung Dis 1997; 1: 44-51.

28. Anand BS, Nanda R, Sachdev GK. Response of tuberculousstricture to anti-tuberculous treatment. Gut 1988; 29:62-69.

29. Balasubramanian R, Ramachandran R, Joseph PE, et al.Interim results of a clinical study of abdominal tuberculosis.Indian $\mathrm{J}$ Tuberc 1989; 36: 117-121.

30. Iseman MD. Treatment of multidrug resistant tuberculosis.N Engl J Med 1993; 329: 784-791.

31. Tahaoglu K, Torun T, Sevim T, et al. The treatment ofmultidrug-resistant tuberculosis in Turkey. N Eng J Med2001; 345: 170-174. 